\title{
Transient Decay of Longitudinal Magnetization in Heterogeneous Spin Systems under Selective Saturation
}

\author{
Hong N. Yeung and SCOTt D. Swanson \\ Department of Radiology, University of Michigan, Ann Arbor, Michigan 48109-0553
}

Received October 22, 1991; revised February 3, 1992

\begin{abstract}
The transient behavior of the longitudinal magnetization of the mobile protons in aqueous heterogeneous materials is investigated both theoretically and experimentally when selective saturation is applied by off-resonance, RF irradiation to the homogeneously broadened, immobile protons which are coupled to the mobile protons through cross relaxation. Analytical solution of this problem is obtained by a generalization of $\mathrm{H}$. C. Torrey's solution to the Bloch equations. Progressive (but indirect) saturation of the magnetization of the mobile protons under this RF irradiation is dynamically monitored using very-small-tipangle sampling pulses. The apparent relaxation rate of heat-denatured egg albumin was measured as a function of frequency offset of the saturating RF with different amplitudes using a new dispersion method modified from a broadband technique recently invented for the rapid acquisition of a cross-relaxation $z$ spectrum. (๑) 1992 Academic Press, Inc.
\end{abstract}

Interpretation of measured spin-lattice relaxation rates in heterogeneous spin systems such as tissues (or gels) is often complicated by the fact that these systems consist of two or more dynamically distinct proton species which are spin coupled to one another. To understand the measured relaxation rates, a physical model is constructed which consists of two thermodynamic reservoirs or spin baths: one contains mobile protons, labeled A, and the other motionally restricted protons, labeled B (1-5). The longitudinal magnetization of each spin species is coupled to the lattice by a characteristic $T_{1}$ process with a rate determined by the molecular dynamics of the particular species. In addition, these magnetizations are coupled by a cross-relaxation term determined by the dipole interaction between, and the chemical exchange of, the A and B spins.

It has been shown that these systems can be studied by observing the steady-state $(6-8)$ and transient $(9,10)$ responses of the A-spin magnetization to the application of a selective saturation of the B-spin reservoir with off-resonance RF irradiation. In medical NMR imaging, a new form of tissue contrast, known as magnetization-transfer contrast (or MTC) $(11-13)$ is achieved by this indirect saturation of water protons through the solid protons by selective saturation. The steady-state solution of a set of truncated, coupled Bloch equations was used by Grad and Bryant (5) to describe the magnitude of indirect saturation of $A$ spins as a function of the frequency of the saturating RF field. The resulting profile of saturation is called a cross relaxation or $z$ spectrum. A more complete version of this solution was recently obtained by $\mathrm{Wu}$ (14), who included the effects of $T_{2}$ relaxation of the A spins and therefore its direct saturation by the irradiating RF, which is significant at low frequency offsets. We 
present here a general solution to the same truncated set of coupled Bloch equations used by Grad and Bryant (5). We also experimentally verify some of the conclusions resulting from the solution, in particular the transient solution, to this problem. Finally, we use the experimental data to provide an estimate of the parameters used in the theory.

\section{THEORY}

The heterogeneous spin system, modeled after a pair of coupled spin baths in the presence of an RF field, can be described (5) by a set of coupled Bloch equations,

$$
\begin{aligned}
& \frac{d M_{\mathrm{A}, \mathrm{B}}^{x}}{\mathrm{~d} \tau}=-\beta_{\mathrm{A}, \mathrm{B}} M_{\mathrm{A}, \mathrm{B}}^{y}-\delta_{\mathrm{A}, \mathrm{B}} M_{\mathrm{A}, \mathrm{B}}^{y} \\
& \frac{d M_{\mathrm{A}, \mathrm{B}}^{y}}{d \tau}=-\beta_{\mathrm{A}, \mathrm{B}} M_{\mathrm{A}, \mathrm{B}}^{y}+\delta_{\mathrm{A}, \mathrm{B}} M_{\mathrm{A}, \mathrm{B}}^{x}-M_{\mathrm{A}, \mathrm{B}}^{z} \\
& \frac{d M_{\mathrm{B}}^{z}}{d \tau}=-\left(\frac{\alpha_{\mathrm{X}}}{f}+\alpha_{\mathrm{B}}\right)\left(M_{\mathrm{B}}^{z}-M_{\mathrm{B}}^{z^{0}}\right)+\alpha_{\mathrm{X}}\left(M_{\mathrm{A}}^{z}-M_{\mathrm{A}}^{z^{0}}\right)+M_{\mathrm{B}}^{y} \\
& \frac{d M_{\mathrm{A}}^{z}}{d \tau}=-\left(\alpha_{\mathrm{X}}+\alpha_{\mathrm{A}}\right)\left(M_{\mathrm{A}}^{z}-M_{\mathrm{A}}^{z^{0}}\right)+\frac{\alpha_{\mathrm{X}}}{f}\left(M_{\mathrm{B}}^{z}-M_{\mathrm{B}}^{z^{0}}\right)+M_{\mathrm{A}}^{y},
\end{aligned}
$$

where $M_{\mathrm{B}, \mathrm{A}}^{z^{0}}$ denote the thermal equilibrium longitudinal magnetization of the A and $B$ spins. The other quantities are dimensionless parameters defined by

$$
\begin{aligned}
\tau=\omega_{1} t, & \alpha_{\mathrm{A}, \mathrm{B}}=1 / \omega_{1} T_{1 \mathrm{~A}, \mathrm{~B}}, \\
\delta_{\mathrm{A}, \mathrm{B}} & =\Delta \omega_{\mathrm{A}, \mathrm{B}} / \gamma \omega_{1}, \quad \Delta \omega_{\mathrm{A}, \mathrm{B}}=\omega-\omega_{\mathrm{A}, \mathrm{B}}^{0}
\end{aligned}
$$

where $\omega_{1}=\gamma B_{1}, T_{1 \mathrm{~A}, \mathrm{~B}}$ and $T_{2 \mathrm{~A}, \mathrm{~B}}$ are the relaxation times of the A and B spins, $r_{\mathrm{X}}$ is the cross-relaxation rate, $\Delta \omega_{\mathrm{A}, \mathrm{B}}$ is the frequency offset of the RF field, and $f$ is molar ratio of the $B$ spins to $\Lambda$ spins. We hereafter make the same assumption as Grad and Bryant (5), who argued that by applying the RF irradiation at a frequency sufficiently far from $\omega_{A}^{0}$, one may partially saturate the homogeneously broadened $B$ spins without directly affecting the A spins. The A spins are of course still affected, but only indirectly through cross relaxation (Eqs. [1e] and [1f]) between the two spin reservoirs. For this reason, it is legitimate to exclude Eqs. [1a] and [1c] from our consideration and set $M_{\mathrm{A}}^{x}=M_{\mathrm{A}}^{y}=0$ in the rest of remaining four equations in Eq. [1]. To further simplify this equation set, we define

$$
u=\frac{M_{\mathrm{B}}^{y}}{M_{\mathrm{B}}^{z^{0}}}, \quad v=\frac{M_{\mathrm{B}}^{y}}{M_{\mathrm{B}}^{z^{0}}}, \quad \text { and } \quad w_{\mathrm{A}, \mathrm{B}}=\frac{M_{\mathrm{A}, \mathrm{B}}^{z^{0}}-M_{\mathrm{A}, \mathrm{B}}^{z}}{2 M_{\mathrm{A}, \mathrm{B}}^{z^{0}}}
$$

and drop the subscript B from the symbols $\beta$ and $\delta$. After these simplifications, the set of equations in [1] is reduced to 


$$
\begin{array}{r}
\frac{d u}{d \tau}+\beta u+\delta v=0 \\
\frac{d v}{d \tau}+\beta v-\delta u+\left(1-2 w_{\mathrm{B}}\right)=0 \\
\frac{d w_{\mathrm{B}}}{d \tau}+\left(\alpha_{\mathrm{B}}+\frac{\alpha_{\mathrm{X}}}{\mathrm{f}}\right) w_{\mathrm{B}}-\frac{\alpha_{\mathrm{X}}}{f} w_{\mathrm{A}}+\frac{v}{2}=0 \\
\frac{d w_{\mathrm{A}}}{d \tau}+\left(\alpha_{\mathrm{A}}+\alpha_{\mathrm{X}}\right) w_{\mathrm{A}}-\alpha_{\mathrm{X}} w_{\mathrm{B}}=0
\end{array}
$$

The steady-state solution to this reduced set of equations has been given by Grad and Bryant (5). To obtain a general solution, we follow an approach much analogous to Torrey's solution (15) to the Bloch equations. The details are given in the Appendix. If we write the components of $u, v, w_{\mathrm{A}}$, and $w_{\mathrm{B}}$ in a column vector, $\mathbf{X}=\left\{u, v, w_{\mathrm{B}}\right.$, $\left.w_{\mathrm{A}}\right)^{\mathrm{T}}$, then the general solution of [4] can be written as

$$
\mathbf{X}=\mathbf{A} e^{-a t}+\mathbf{B} e^{-b \tau}+\mathbf{C} e^{-c \tau} \cos (s \tau)+\frac{\mathbf{D}}{s} e^{-c \tau} \sin (s \tau)+\mathbf{E},
$$

where $\mathbf{A}, \mathbf{B}, \mathbf{C}, \mathbf{D}$, and $\mathbf{E}$ are column vectors and, along with the constants $a, b, c$, and $s$, are functions of $\alpha_{\mathrm{A}, \mathrm{B}}, \beta$, and $\delta$. The first four terms of [5] give the transient effects and the last term gives the steady-state effects. The observable steady-state longitudinal component of the mobile protons $w_{\mathrm{A}}(\infty)$ as given by the fourth component of $\mathbf{E}$ in the Appendix (Eq. [A10]),

$$
w_{\mathrm{A}}(\infty)=E_{4}=\frac{1}{2} \frac{\beta \alpha_{\mathrm{X}}}{\left(\beta^{2}+\delta^{2}\right)\left\{\alpha_{\mathrm{B}}\left(\alpha_{\mathrm{A}}+\alpha_{\mathrm{X}}\right)+\alpha_{\mathrm{A}} \alpha_{\mathrm{X}} / f\right\}+\alpha_{\mathrm{B}}\left(\alpha_{\mathrm{A}}+\alpha_{\mathrm{X}}\right)},
$$

must of course agree, as in fact it does, with the steady-state solution of $w_{\mathrm{A}}$ given by Grad and Bryant (5). The constants, $a, b, c$, and $s$ are the same for all components of $\mathbf{X}$ and, along with the vector $\mathbf{E}$, are independent of the initial conditions. The coefficient vectors $\mathbf{A}, \mathbf{B}, \mathbf{C}$, and $\mathbf{D}$, however, depend on the initial conditions as defined by the two vectors $\mathbf{X}(0)$ and $\dot{\mathbf{X}}(0)$. The factors $a, b, c$, and $s$ and the vectors $\mathbf{A}, \mathbf{B}, \mathbf{C}, \mathbf{D}$, and $\mathbf{E}$ are all evaluated implicitly in the Appendix.

Although Eq. [5] can be expressed only implicitly in terms of the fundamental constants which describe the spin-bath model, it is illuminating to examine by numerical evaluation the conditions of validity of the assumptions made, implicitly or explicitly, in the analysis of relaxation-time experiments in heterogeneous systems. For instance, in the IRSAT experiments by Grad et al. (9) as mentioned above, apparent longitudinal relaxation rates were measured by a standard inversion-recovery method with selective saturation applied throughout the idle periods of the pulse sequence. Under this continuous irradiation, the magnetization of the B spins is assumed to be completely saturated. The condition of complete saturation is not essential; what is essential is that the $B$ magnetization reaches a steady state. The time derivatives of this magnetization are therefore zero and only one equation [4d] which describes the longitudinal component of the A spin remains. The solution of this equation yields an apparent relaxation rate for the $\mathrm{A}$ spins which equals $1 / T_{1 \mathrm{~A}}+r_{\mathrm{X}}$, or in our notation 
$\omega_{1}\left(\alpha_{\mathrm{A}}+\alpha_{\mathrm{X}}\right)$. In light of the general solution [5], we can now assess the conditions, if there are any, under which the above simplified result is valid. We shall return to this point under Results and Interpretation.

Three issues need to be considered. First, since Eq. [5] consists of three rate constants, the observed decay is in general not a single exponential. It is so only if one of these constants, say $a$, is much smaller than the others, and even then, only after the fast transients decay to near zero. For most systems, these conditions are satisfied and monoexponential decays or recoveries are observed (after a short delay from an inversion pulse, typically $10 \mathrm{~ms}$ ). To obtain an explicit solution, we must therefore identify $a$ in terms of the parameters of the spin-bath model. We make no attempt to do so here. Second, from the analysis leading to Eq. [5], we know that the rate constants $a, b, c$, and $s$ are independent of the initial conditions. As a consequence, the apparent relaxation rate measured by monitoring the longitudinal time development of $M_{\mathrm{A}}^{z}$ must also be independent of the initial preparation of the spin systems. Since the apparent relaxation rate is independent of initial preparation, it follows that presaturation of the $B$ spins is not required to measure the apparent relaxation rate in the presence of off-resonance irradiation as has previously been done (9). In the experimental section we describe measurement of the apparent relaxation rate $R_{1}^{\text {app }}$ of heatdenatured egg albumin with off-resonance irradiation as a function of frequency offset (at different saturating RF strengths), with and without presaturation of the spin system, and verify that $R_{1}^{\mathrm{app}}$ is indeed the same regardless of the preparation. Finally, using heat-denatured egg albumin as an example, we examine the condition under which, if any, $R_{1}^{\text {app }}$ approaches the limiting value $1 / T_{1 \mathrm{~A}}+r_{\mathrm{X}}$.

\section{EXPERIMENTAL METHOD AND MATERIALS}

The longitudinal relaxation rate with off-resonance saturation was measured for all off-resonance frequencies with a technique much like the broadband technique used to obtain the cross-relaxation NMR spectrum (16). Briefly, the broadband method achieves all conditions of off-resonance saturation for the cross-relaxation experiment by applying a gradient and a saturation RF pulse to the sample concurrently. The concurrent application of a gradient and an RF pulse can be called "broadband saturation." The current work deals with the transient effects of cross relaxation. We verify experimentally that the rate at which the magnetization reaches steady state with the application of off-resonance saturation does not depend on the initial conditions of the magnetization of the solid or liquid spins. Relaxation rates were measured by inversion recovery (IR). To follow the time course of the relaxation curve dynamically, we used Look and Locker's technique (17) with small-tip-angle monitoring pulses. The pulse sequences are shown schematically in Fig. 1. The experiments were performed in a General Electric $2.0 \mathrm{~T}, 33 \mathrm{~cm}$ bore CSI system. The sample used was a tube containing heat-denatured hen-egg albumin.

Two types of IR experiment (Fig. 1) were performed together with this broadband progressive saturation technique: (a) with a $600 \mathrm{~ms}$ presaturation pulse and RF irradiation applied continuously following the inversion pulse (i.e., a broadband analog of IRSAT) and (b) same as (a) but without the presaturation pulse. In addition to these experiments, we also acquired a $T_{\text {INS }}$ data set (c) with no saturation applied before or after the inversion pulse (i.e., a conventional IR experiment) and an $M_{0}^{\prime}$ 

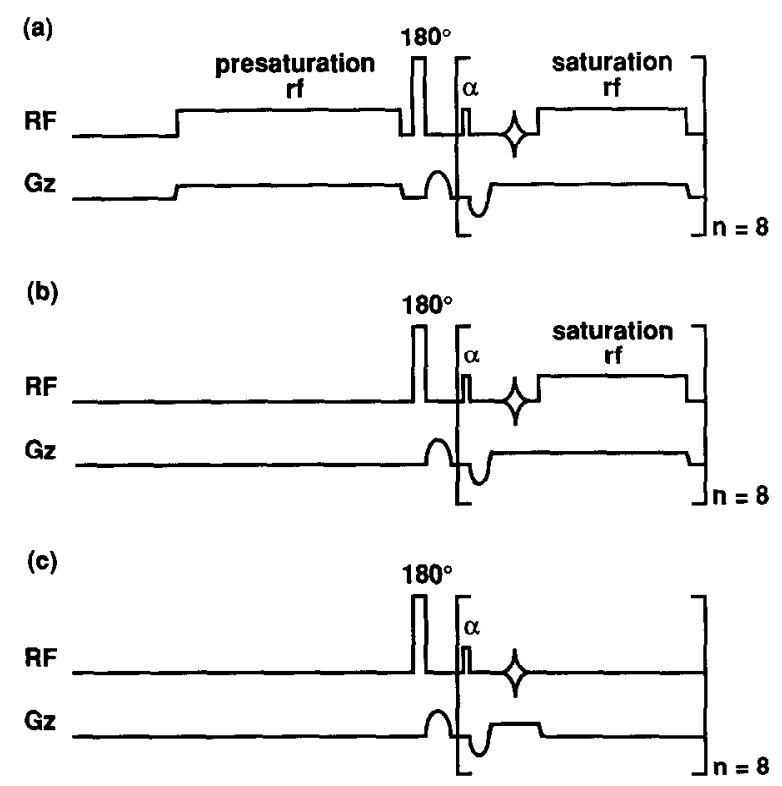

FIG. 1. Schematic diagram of the pulse sequences used in the experiments: Look and Locker inversion recovery (a) with broadband saturation (or dispersive $T_{1 \text { sat }}$ ) experiments with presaturation, (b) same as (a) except with no presaturation, and (c) with neither a pre- nor a postsaturation RF pulse.

data set (d) with neither RF irradiation nor inversion pulse. Data set (d) was used to normalize the IR data and compute the ratios $M_{\mathrm{s}} / M_{0}^{\prime}$, where $M_{0}^{\prime}$ is the steady-state magnetization with no saturation,

$$
M_{0}^{\prime}=M_{0} \frac{1-e^{-t_{\mathrm{d}} / T_{\mathrm{INS}}}}{1-\cos \alpha e^{-t_{\mathrm{d}} / T_{\mathrm{NSS}}}},
$$

with $\alpha$ being the tip angle and $t_{\mathrm{d}}$ the interpulse interval of the Look and Locker monitoring pulses.

Phase-sensitive inversion-recovery data were obtained by dividing the IR data sets by the $M_{0}^{\prime}$ data. Phase anomaly in the IR data is removed by cancellation, because the phasing of the data (except for the phase inversion caused by the $180^{\circ}$ pulse) remains constant regardless of whether the broadband saturation RF and/or the inversion pulse are applied. With presaturation off but broadband saturation on, the excursion of the magnetization will be from $-M_{0}$ to $M_{\text {sat }}$. Dividing these data by $M_{0}^{\prime}$ scales them from $-M_{0} / M_{0}^{\prime}$ to $M_{\text {sat }} / M_{0}^{\prime}$. We point out here that the dynamic range of this experiment is greater than that of the IRSAT experiment, where magnetization ranges from $-M_{\text {sat }}$ to $M_{\text {sat }}$ (or $-M_{\text {sat }} / M_{0}^{\prime}$ to $M_{\text {sat }} / M_{0}^{\prime}$ after scaling).

Assuming monoexponential decay, the approach to saturation of the longitudinal component will follow the empirical relation

$$
M_{z}(t)=A^{\prime}+B^{\prime} e^{-k_{\tau}},
$$

where $A^{\prime}=M_{\text {sat }} / M_{0}^{\prime}$ and $B^{\prime}=\left[M_{z}(0)-M_{\text {sat }}\right] / M_{0}^{\prime}$, with $M_{\text {sat }}$ being the steady state 
and $M_{z}(0)$ the initial longitudinal magnetization. The rate constant $\kappa$ is related to the apparent relaxation rate $R_{1}^{\text {app }}$ by the relation

$$
\kappa=R_{1}^{\mathrm{app}}-\frac{\ln (\cos \alpha)}{t_{\mathrm{d}}} .
$$

The tip angles $\alpha$ and time delays $t_{\mathrm{d}}$ used in all our experiments were $15^{\circ}$ and $300 \mathrm{~ms}$, respectively. The parameters $\kappa, A^{\prime}$, and $B^{\prime}$ were obtained by a nonlinear least-squares fitting program in the system software of the GE-CSI system. The apparent relaxation rate $R_{1}^{\text {app }}$ was obtained after the tip-angle correction of $\kappa$ from Eq. [9]. The steadystate magnetization, $M_{\text {sat }}$, is simply equal to $A^{\prime} M_{0}^{\prime}$.

\section{RESULTS AND INTERPRETATION}

The apparent relaxation rates of the water protons in heat-denatured egg albumin measured as a function of frequency offset with and without presaturation are shown in Fig. 2. These data show that, as predicted under Theory, the apparent relaxation rate is not a function of the initial conditions of the spin system.

The experimentally measured and theoretically fitted relaxation rates and steady-state magnetizations are plotted as a function of the offset frequency of the saturation $B_{1}$ field

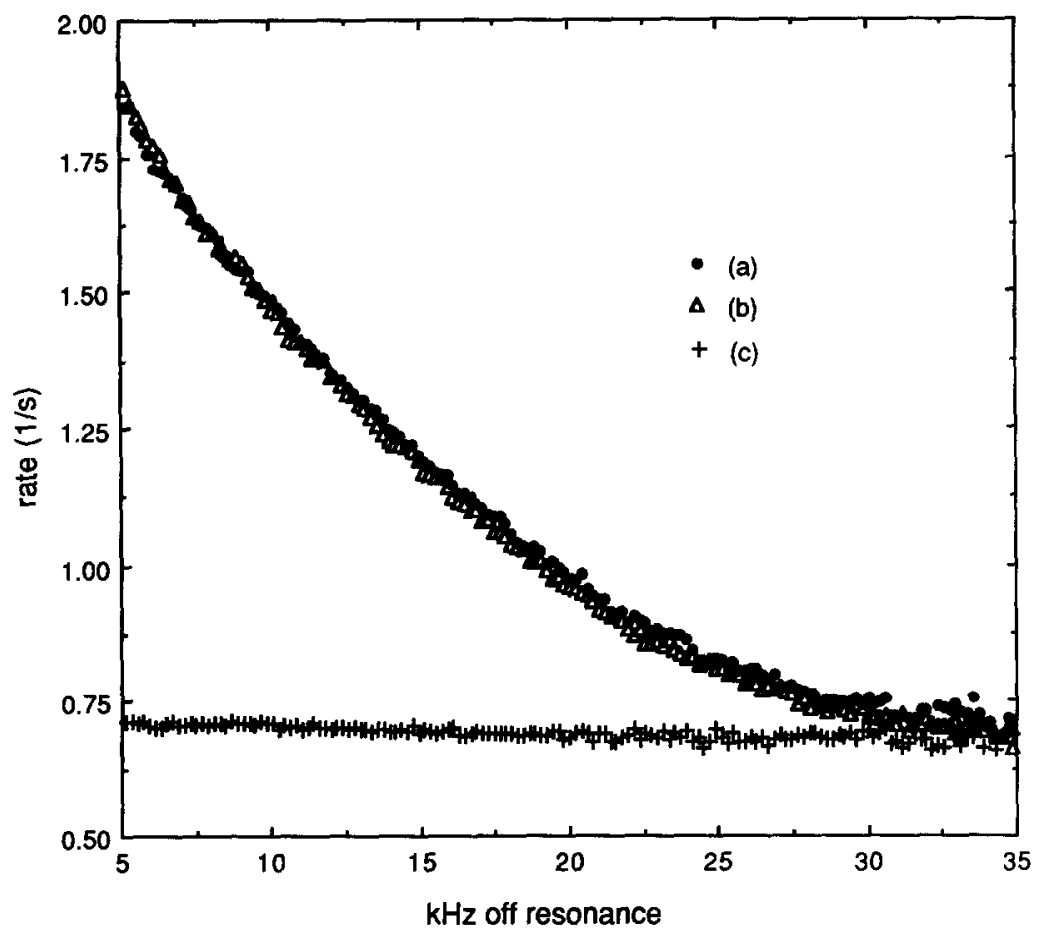

FIG. 2. Apparent relaxation rates with off-resonance RF saturation vs frequency offsets of the saturating RF; the resulting plots are from data sets obtained by inversion recovery (a) with presaturation, (b) without presaturation, and (c) using the conventional method with neither presaturation nor postsaturation. 
for different levels of $B_{1}$ in Fig. 3. The fitted curves were obtained by the numerical evaluation (using Mathematica in a Macintosh computer) of $a$ and the fourth component of $\mathbf{E}$ as a function of $\delta$ at five different values of $\omega_{1}$ with a consistent set of adjusted parameters of $T_{1 \mathrm{~A}}, T_{1 \mathrm{~B}}, T_{2 \mathrm{~B}}, r_{\mathrm{X}}$, and $f$. (The rate constant $a$ is by convention the smallest negative root of the quartic equation $\Delta(x)=0$; the fourth-degree polynomial $\Delta(x)$ is given in Eq. [A3] in the Appendix.) The parameter set with the best fit is $\left\{1 / T_{1 \mathrm{~A}}=0.33\right.$ $\mathrm{s}^{-1}, 1 / T_{\mathrm{BB}}=5.24 \mathrm{~s}^{-1}, T_{2 \mathrm{~B}}=60 \mu \mathrm{s}, r_{\mathrm{X}}=3.0 \mathrm{~s}^{-1}$, and $\left.f=0.09\right\}$. Of these five parameters, the only adjustment we allowed ourselves to make is the values of $r_{\mathrm{X}}$ and $T_{2 \mathrm{~B}}$ (see below). The other three parameters were chosen on the basis of the following criteria: $T_{1 \mathrm{~A}}$ is the $T_{1}$ of pure water, which at room temperature is approximately $3 \mathrm{~s}$, the molar ratio $f$ is an estimate based on the wet/dry weight measurement performed on a similarly prepared but different heat-denatured egg albumin sample (18), and $1 / T_{1 \mathrm{~B}}$, or $r_{\mathrm{B}}$, is fixed from the constraint $(10)$ that the measured $1 / T_{1 \mathrm{NS}}$ must equal $\lambda_{-}$, where $\lambda_{-}$is the smaller eigenvalue of the binary-coupled longitudinal component equations in the absence of RF irradiation; i.e.,

$$
\begin{aligned}
\lambda_{-}=\frac{1}{T_{\mathrm{INS}}}=\frac{1}{2}\left\{r_{\mathrm{A}}+r_{\mathrm{B}}+r_{\mathrm{X}}\left(1+\frac{1}{f}\right)\right\} & \\
& -\frac{1}{2} \sqrt{\left\{r_{\mathrm{\Lambda}}-r_{\mathrm{B}}+r_{\mathrm{X}}\left(1-\frac{1}{f}\right)\right\}^{2}+\frac{4 r_{\mathrm{X}}^{2}}{f}} .
\end{aligned}
$$

Because of the assumption made in Eq. [4], the fitting of the data is not expected to be good at low frequency offsets. At higher offsets the fitting is reasonably good for the two cases when the saturating RF level is low. When the RF level is high, the fitting is still good up to $\sim 20 \mathrm{kHz}$, beyond which the experimental values of $R_{1}^{\text {app }}$ become consistently low compared to the calculated values. The fitting of the steady-state $M_{\text {sat }}$, while reasonably well in overall amplitude, shows a distinct difference in the solid lineshape. Both of these discrepancies can be explained by the inherent assumption made in describing the heterogeneous spin system by the Bloch equations such as Eqs. [1b, 1d] and [4a, 4b]. These equations automatically imply a Lorentzian lineshape in the solid component, which is most likely not true. For this reason, the estimate of the parameter $T_{2 \mathrm{~B}}$ is purely empirical. We did make the attempt to estimate $T_{2 \mathrm{~B}}$ by the measurement of $z$-spectrum linewidth as a function of field strength of the saturating $\operatorname{RF}(5,16)$, but the measured value ( $\sim 20 \mu \mathrm{s}$ ) was too low to yield a consistently good fit to all the available data. In summary, with this inadequacy notwithstanding, the binary spin-bath model gives a reasonable account of most of our experimental results.

Our final objective is to determine the conditions, if they exist at all, under which the simple interpretation of the measured apparent relaxation rate reached by Grad et al. (9), $R_{1}^{\text {app }}=1 / T_{1 \mathrm{~A}}+r_{\mathrm{X}}$, can be made by applying constant off-resonant saturation. We use the fitting parameters of the heat-denatured egg albumin as an example except for the molar ratio $f$, which we shall assume to be variable. Figure 4 shows the threedimensional plot of the rate constant $a$ as a function of frequency offset $\Delta \omega$ of the saturating RF and the molar ratio $f$. It is clear from Fig. 4 that the simple interpretation $R_{1}^{\text {app }}=1 / T_{1 \mathrm{~A}}+r_{\mathrm{X}}$ is in general not valid, but may be a good approximation when $f$ is sufficiently large $(>0.4)$ and $\Delta \omega$ is sufficiently small $(<10 \mathrm{kHz})$. 


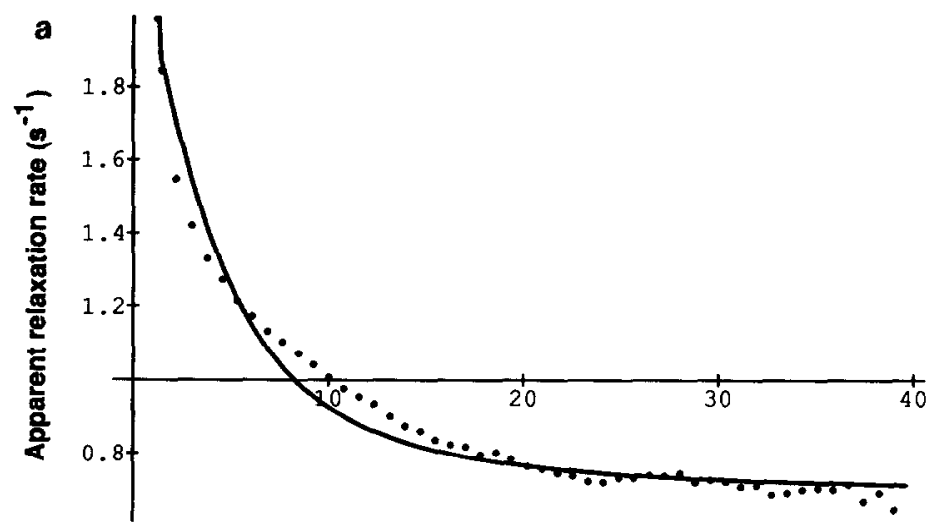

Frequency offset $(\mathrm{KHz})$

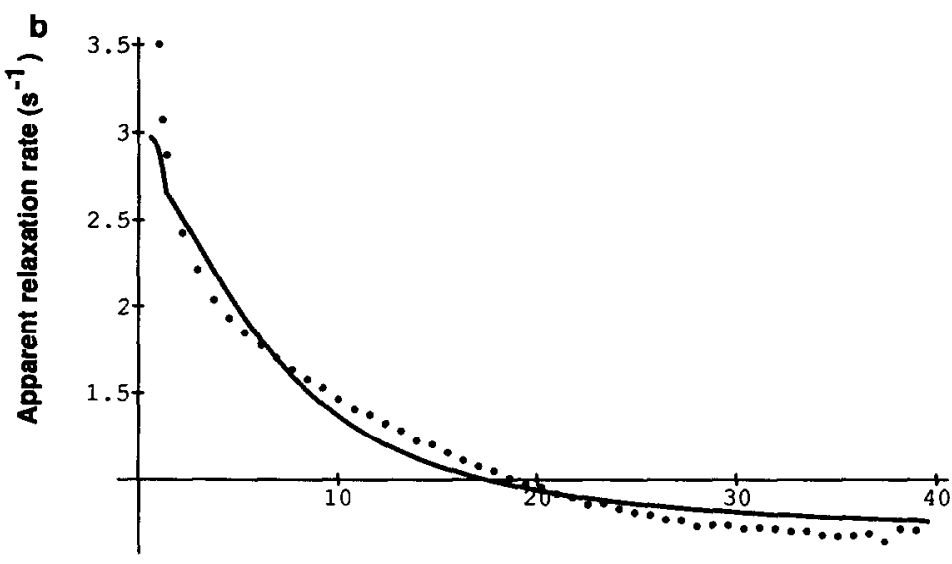

Frequency offset (KHz)

FIG. 3. Fitting of the broadband $T_{1 \text { sat }}$ and steady-state magnetization data to theoretical calculations. The parameter set that fits all the data "best" is $\left\{1 / T_{1 \mathrm{~A}}=0.33 \mathrm{~s}^{-1}, 1 / T_{1 \mathrm{~B}}=5.2 \mathrm{~s}^{-1}, T_{2 \mathrm{~B}}=60 \mu \mathrm{s}, r_{\mathrm{X}}=3.0 \mathrm{~s}^{-1}\right.$, and $f=0.09\}$. (a) $T_{1 \text { sat }}$ data at $\omega_{1}=1086 \mathrm{rad} / \mathrm{s}$; (b) $T_{1 \text { sat }}$ data at $\omega_{1}=2026 \mathrm{rad} / \mathrm{s}$; (c) $T_{1 \text { sat }}$ data at $\omega_{1}$ $=2898 \mathrm{rad} / \mathrm{s}$; (d) $T_{1 \text { sat }}$ data at $\omega_{\mathrm{t}}=3675 \mathrm{rad} / \mathrm{s}$; (e) $T_{1 \text { sat }}$ data at $\omega_{1}=4241 \mathrm{rad} / \mathrm{s}$; (f) steady-state signal data at $\omega_{1}=2026 \mathrm{rad} / \mathrm{s}$.

\section{SUMMARY AND CONCLUSIONS}

To summarize, we have provided a general solution to the generalized Bloch equations of the heterogeneous spin system as modeled by the binary-coupled spin baths. We have presented theoretical and experimental evidence that shows that the simple relation reached by Grad et al. in the IRSAT experiments is in general invalid but is approachable under special circumstances such as when $f$ is large and the offset frequency of the saturation $\mathrm{RF}$ is small. We have also shown both in theory and in experiment that the presaturation event in the IRSAT experiment is superfluous and that by measuring the apparent relaxation rate as a function of frequency offset and amplitude of the RF saturation, it is 


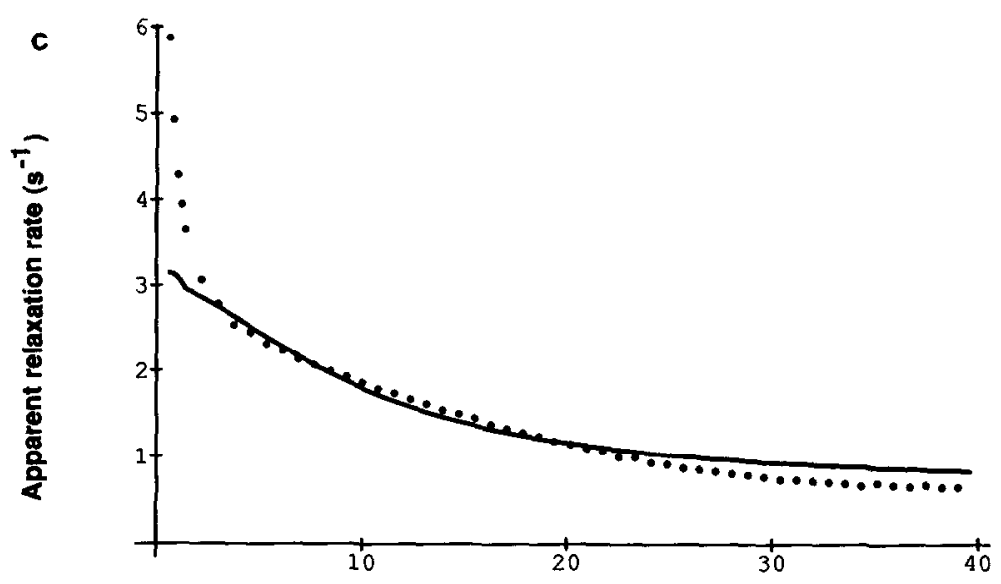

Frequency offset (KHz)

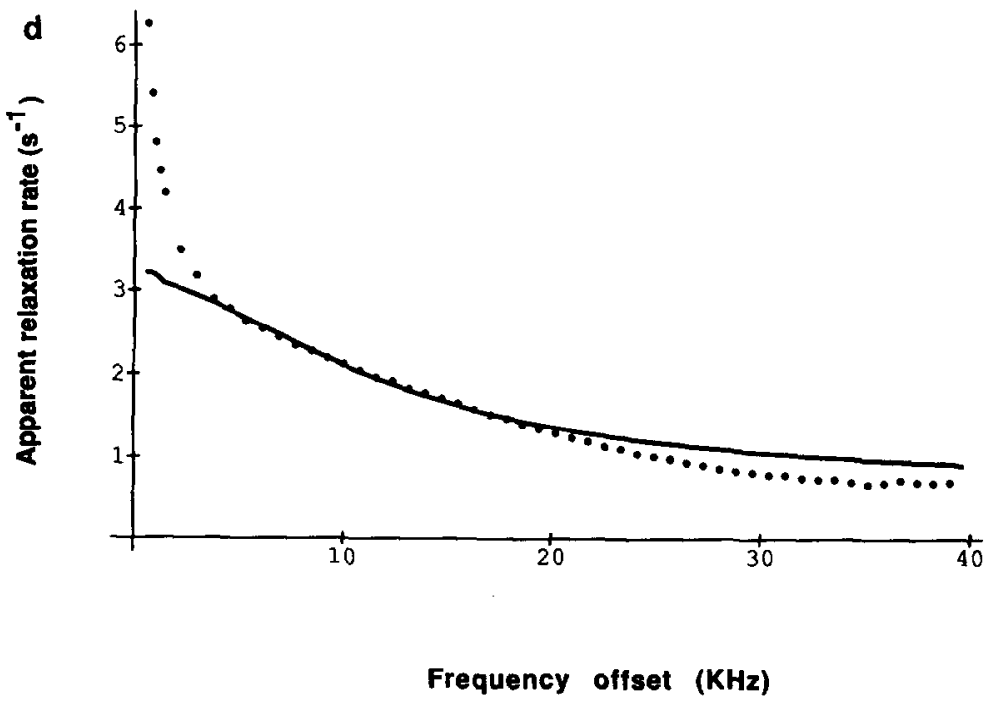

FIG. 3-Continued

possible, though not always practical, to deduce the whole set of model parameters which satisfactorily describe the spin baths.

\section{APPENDIX}

The method given herc for the general solution of the coupled equations in [4] is a straight generalization of the procedure given by Torrey (15) in his appendix for the general solution of the Bloch equations. For this reason, the same symbols will be used as those he used for quantities that have identical or equivalent physical meanings. 


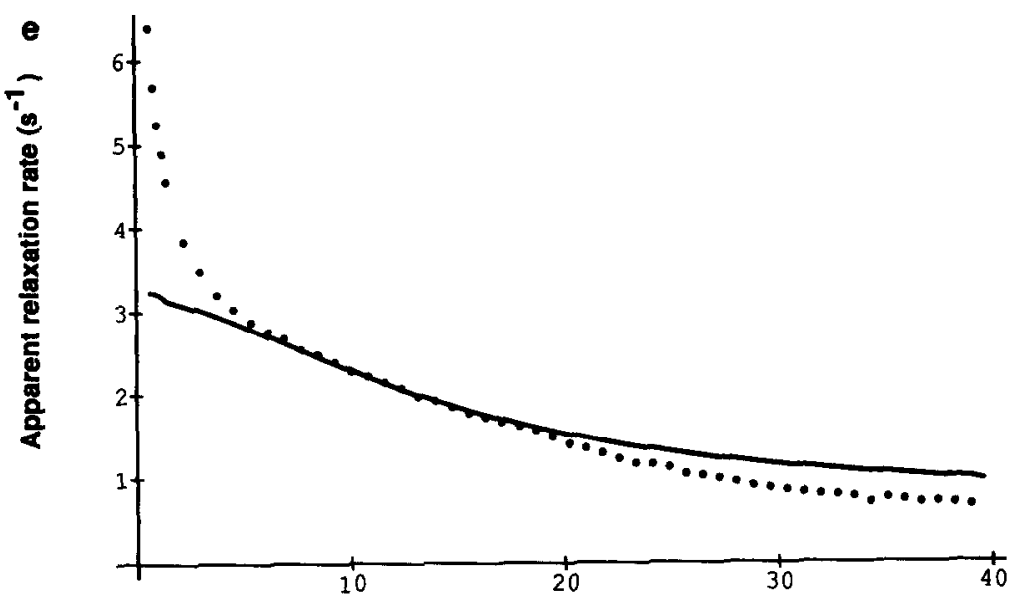

Frequency offset (KHz)

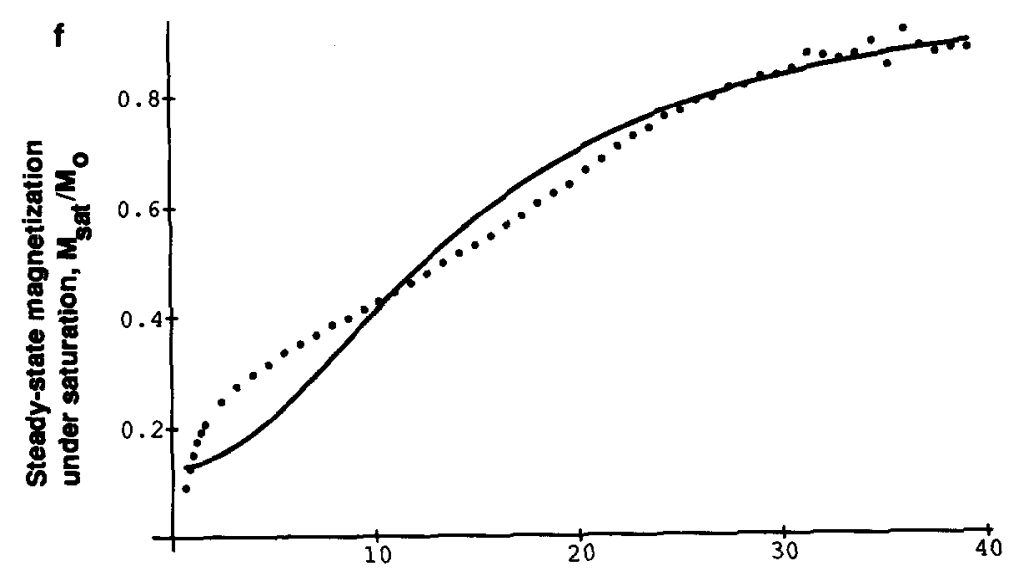

Frequency offset (KHz)

FIG. 3-Continued

The Laplace transforms of Eq. [4] in the text are

$$
\begin{aligned}
(p+\beta) \tilde{u}+\delta \tilde{v} & =u_{0} \\
-\delta \tilde{u}+(p+\beta) \tilde{v}-2 \tilde{w}_{\mathrm{B}} & =v_{0}-\frac{1}{p} \\
\frac{\tilde{v}}{2}+\left(p+\frac{\alpha_{\mathrm{X}}}{f}+\alpha_{\mathrm{B}}\right) \tilde{w}_{\mathrm{B}}-\frac{\alpha_{\mathrm{X}}}{f} \tilde{w}_{\mathrm{A}} & =w_{\mathrm{B} 0} \\
-\alpha_{\mathrm{X}} \tilde{w}_{\mathrm{B}}+\left(p+\alpha_{\mathrm{A}}+\alpha_{\mathrm{X}}\right) \tilde{w}_{\mathrm{A}} & =w_{\mathrm{A} 0},
\end{aligned}
$$


where $\tilde{u}, \tilde{v}, \tilde{w}_{\mathrm{B}}$, and $\tilde{w}_{\mathrm{A}}$ are the transforms and $u_{0}, v_{0}, w_{\mathrm{B} 0}$, and $w_{\mathrm{A} 0}$ the initial values of $u, v, w_{\mathrm{B}}$, and $w_{\mathrm{A}}$, respectively. Because of the algebraic complexity for an arbitrary initial condition, we shall confine our attention to the cases where only the longitudinal magnetizations of the two spin species are allowed to be arbitrary; the initial transverse magnetization components of the $\mathrm{B}$ spins are assumed to be 0 . So if we let $w_{\mathrm{A} 0}=\eta$ and $w_{\mathrm{B} 0}=\lambda, 0 \leqslant(\lambda, \eta) \leqslant 1$, then the initial vector $\mathbf{X}(0)=\{0,0, \lambda, \eta\}^{\mathrm{T}}$ and the solutions of $[\mathrm{Al}]$ for this initial condition are

$$
\begin{aligned}
& p \Delta(p) \tilde{u}=\left[\delta\left\{\alpha_{\mathrm{A}} \alpha_{\mathrm{X}} / f+\alpha_{\mathrm{B}}\left(\alpha_{\mathrm{A}}+\alpha_{\mathrm{X}}\right)+p\left[\alpha_{\mathrm{A}}+\alpha_{\mathbf{B}}+\alpha_{\mathbf{X}}(1+1 / f)\right]+p^{2}\right\}\right] \\
& -2 \delta p\left\{\lambda\left(p+\alpha_{\mathrm{A}}+\alpha_{\mathrm{X}}\right)+\eta\left(\alpha_{\mathrm{X}} / f\right)\right\} \\
& p \Delta(p) \tilde{v}=\left[(\beta+p)\left\{\alpha_{\mathrm{A}} \alpha_{\mathrm{X}} / f+\alpha_{\mathrm{B}}\left(\alpha_{\mathrm{A}}+\alpha_{\mathrm{X}}\right)+p\left[\alpha_{\mathrm{A}}+\alpha_{\mathrm{B}}+\alpha_{\mathbf{X}}(1+1 / f)\right]+p^{2}\right\}\right] \\
& +2 p\left[\lambda\left\{(\beta+p)\left(p+\alpha_{\mathrm{A}}+\alpha_{\mathrm{X}}\right)\right\}+\eta\left(\alpha_{\mathrm{X}} / f\right)(\beta+p)\right] \\
& p \Delta(p) \tilde{w}_{\mathrm{B}}=\left[(\beta+p)\left(\alpha_{\mathrm{A}}+\alpha_{\mathrm{X}}+p\right) / 2\right]+p\left[\lambda \left\{\left(\beta^{2}+\delta^{2}\right)\left(p+\alpha_{\mathrm{A}}+\alpha_{\mathrm{X}}\right)\right.\right. \\
& \left.+2 \beta p\left(\alpha_{\mathrm{A}}+\alpha_{\mathrm{X}}\right)+p^{2}\left(2 \beta+\alpha_{\mathrm{A}}+\alpha_{\mathrm{X}}\right)+p^{3}\right\} \\
& +(\eta / f)\left[\alpha_{X}\left(\beta^{2}+\delta^{2}\right)+\alpha_{X} p(p+2 \beta)\right] \\
& p \Delta(p) \tilde{w}_{\mathrm{A}}=\alpha_{\mathrm{X}}[(\beta+p) / 2]+p\left[\lambda \alpha_{\mathrm{X}}\left\{\left(\beta^{2}+\delta^{2}\right)+2 \beta p+p^{2}\right\}\right. \\
& +\eta\left\{\beta+\left(\beta^{2}+\delta^{2}\right)\left(\alpha_{\mathrm{B}}+\alpha_{\mathrm{X}} / f\right)+\left[1+2 \beta\left(\alpha_{\mathrm{B}}+\alpha_{\mathrm{X}} / f\right)\right.\right. \\
& \left.\left.\left.+\left(\beta^{2}+\delta^{2}\right)\right]+p^{2}\left(\alpha_{\mathrm{B}}+\alpha_{\mathrm{X}} / f+2 \beta\right)+p^{3}\right\}\right],
\end{aligned}
$$

where

$$
\begin{aligned}
& \Delta(p)=p^{4}+p^{3}\left[\alpha_{\mathrm{A}}+\alpha_{\mathrm{B}}+\alpha_{\mathrm{X}}(1+1 / f)+2 \beta\right] \\
& \quad+p^{2}\left[\left(\beta^{2}+\delta^{2}\right)+\left(\alpha_{\mathrm{A}}+\alpha_{\mathrm{X}}\right)\left(2 \beta+\alpha_{\mathrm{B}}\right)+\left(1+2 \beta \alpha_{\mathrm{B}}\right)+\left(\alpha_{\mathrm{X}} / f\right)\left(\alpha_{\mathrm{A}}+2 \beta\right)\right] \\
& \quad+p\left[\left(\beta^{2}+\delta^{2}\right)\left[\alpha_{\mathrm{A}}+\alpha_{\mathrm{B}}+\alpha_{\mathrm{X}}(1+1 / f)\right]+\left(\alpha_{\mathrm{A}}+\alpha_{\mathrm{X}}\right)\left(1+2 \beta \alpha_{\mathrm{B}}\right)\right. \\
& \left.\quad+\beta\left(1+2 \alpha_{\mathrm{A}} \alpha_{\mathrm{X}} / f\right)\right]+\left(\beta^{2}+\delta^{2}\right)\left[\alpha_{\mathrm{B}}\left(\alpha_{\mathrm{A}}+\alpha_{\mathrm{X}}\right)+\alpha_{\mathrm{A}} \alpha_{\mathrm{X}} / f\right]+\beta\left(\alpha_{\mathrm{A}}+\alpha_{\mathrm{X}}\right)
\end{aligned}
$$

is the determinant of the coefficients in [A1]. Now we can write

$$
\check{\mathbf{X}}(p)=\mathbf{g}(p) / p \Delta(p),
$$

where

$$
\mathbf{g}(p) \equiv\left[\begin{array}{c}
{\left[\delta \left\{\alpha_{\mathrm{A}} \alpha_{\mathrm{X}} / f+\alpha_{\mathrm{B}}\left(\alpha_{\mathrm{A}}+\alpha_{\mathrm{X}}\right)+p\left[\alpha_{\mathrm{A}}+\alpha_{\mathrm{B}}\right.\right.\right.} \\
\left.\left.\left.+\alpha_{\mathrm{X}}(1+1 / f)\right]+p^{2}\right\}\right]+p\left(h_{1} \lambda+k_{1} \eta\right) \\
(\beta+p)\left\{\alpha_{\mathrm{A}} \alpha_{\mathrm{X}} / f+\alpha_{\mathrm{B}}\left(\alpha_{\mathrm{A}}+\alpha_{\mathrm{X}}\right)+p\left[\alpha_{\mathrm{A}}+\alpha_{\mathrm{B}}\right.\right. \\
\left.+\alpha_{\mathrm{X}}(1+1 / f)\right]+p\left(h_{2} \lambda+k_{2} \eta\right) \\
{\left[(\beta+p)\left(\alpha_{\mathrm{A}}+\alpha_{\mathrm{X}}+p\right) / 2\right]+p\left(h_{3} \lambda+k_{3} \eta\right)} \\
\alpha_{\mathrm{X}}[(\beta+p) / 2]+p\left(h_{4} \lambda+k_{4} \eta\right)
\end{array}\right]
$$




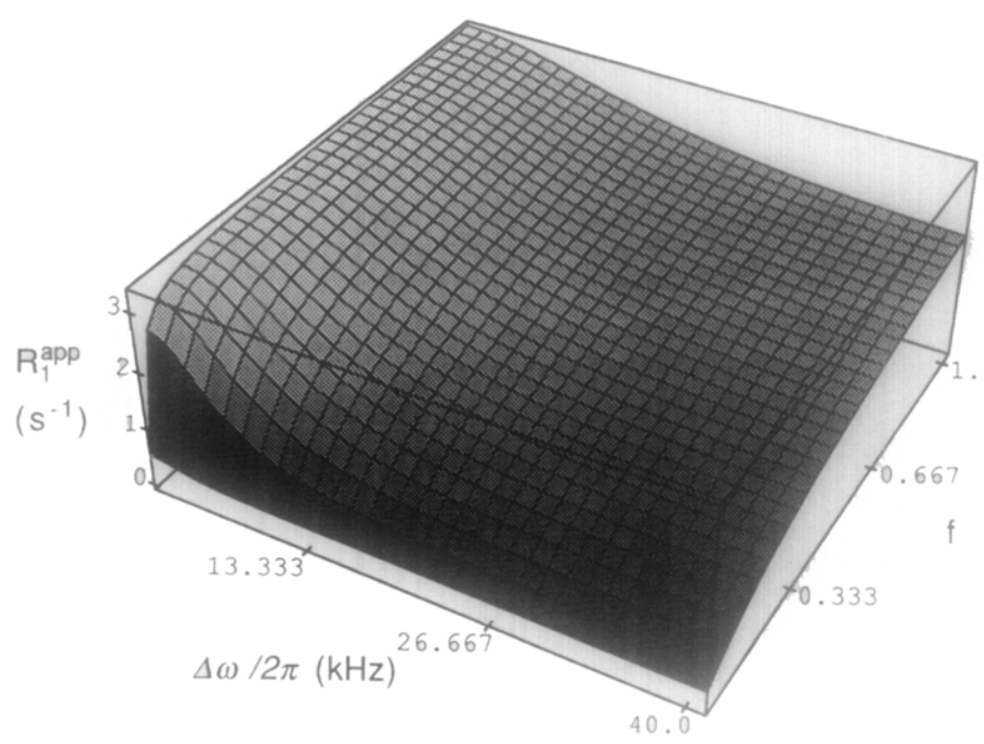

FIG. 4. Theoretical apparent relaxation rate of a hypothetical substance (with the same parameters as the heat-denatured egg albumin except for the molar ratio $f$, which is allowed to vary) as a function of frequency offset of the irradiating RF and $f$.

with

$$
\begin{aligned}
& h_{1}=-2 \delta\left(p+\alpha_{\mathrm{A}}+\alpha_{\mathrm{X}}\right), \quad k_{1}=-2 \delta \frac{\alpha_{\mathrm{X}}}{f} \\
& h_{2}=2(\beta+p)\left(p+\alpha_{\mathrm{A}}+\alpha_{\mathrm{X}}\right), \quad k_{2}=2 \frac{\alpha_{\mathrm{X}}}{f}(\beta+p) \\
& h_{3}=\left(\beta^{2}+\delta^{2}\right)\left(p+\alpha_{\mathrm{A}}+\alpha_{\mathrm{X}}\right)+2 \beta p\left(\alpha_{\mathrm{A}}+\alpha_{\mathrm{X}}\right)+p^{2}\left(2 \beta+\alpha_{\mathrm{A}}+\alpha_{\mathrm{X}}\right)+p^{3} \\
& f k_{3}=h_{4}=\alpha_{\mathrm{X}}\left\{\left(\beta^{2}+\delta^{2}\right)+p(p+2 \beta)\right\} \\
& k_{4}=\beta+\left(\beta^{2}+\delta^{2}\right)\left(\alpha_{\mathrm{B}}+\frac{\alpha_{\mathrm{X}}}{f}+p\right) \\
& \quad+p\left\{1+2 \beta\left(\alpha_{\mathrm{B}}+\frac{\alpha_{\mathrm{X}}}{f}\right)\right\}+p^{2}\left\{\alpha_{\mathrm{B}}+\frac{\alpha_{\mathrm{X}}}{f}+2 \beta\right\}+p^{3} . \quad \text {. AS }
\end{aligned}
$$

Physical reality dictates that the quartic equation $\Delta(p)=0$ has at least two negative roots. Let these roots be $-a,-b$; then $\Delta(p)$ can be factored into

$$
\Delta(p)=(p+a)(p+b)\left\{(p+c)^{2}+s^{2}\right\} .
$$

Equation [A4] can now be expanded in partial fractions:

$$
\tilde{\mathbf{X}}(p)=\frac{\mathbf{A}}{p+a}+\frac{\mathbf{B}}{p+b}+\frac{\mathbf{C} p+\mathbf{D}}{(p+c)^{2}+s^{2}}+\frac{\mathbf{E}}{p} .
$$

The inverse Laplace transform of [A7] yields the desired general solution: 


$$
\mathbf{X}(\tau)=\mathbf{A} e^{-a \tau}+\mathbf{B} e^{-b \tau}+\mathbf{C} e^{-c \tau} \cos (s \tau)+\frac{\mathbf{D}}{s} e^{-c \tau} \sin (s \tau)+\mathbf{E}
$$

It is clcar from [A8] that $\mathbf{E}$ yiclds the steady-state solutions while $\mathbf{A}, \mathbf{B}, \mathbf{C}$, and $\mathbf{D}$ determine the transient behavior of magnetization. The constants $c$ and $s$ are determined by equating coefficients of the terms in same powers in $p$ of the identity

$$
\Delta(p) \equiv(p+a)(p+b)\left\{(p+c)^{2}+s^{2}\right\}
$$

or, from [A3] we obtain

$$
\begin{aligned}
& c=\frac{\alpha_{\mathrm{B}}+\alpha_{\mathrm{A}}+\alpha_{\mathrm{X}}(1+1 / f)+2 \beta-(a+b)}{2} \\
& s=\sqrt{\frac{\left(\beta^{2}+\delta^{2}\right)\left[\alpha_{\mathrm{B}}\left(\alpha_{\mathrm{A}}+\alpha_{\mathrm{X}}\right)+\alpha_{\mathrm{A}} \alpha_{\mathrm{X}} / f\right]+\beta\left(\alpha_{\mathrm{A}}+\alpha_{\mathrm{X}}\right)}{a b}-c^{2}} .
\end{aligned}
$$

The coefficient vectors A, B, and F are given, from [ A7] and [A4], by the following relations:

$$
\begin{aligned}
& \mathbf{E}=\lim _{p \rightarrow 0} p \tilde{\mathbf{X}}(p)=\frac{\mathbf{g}(0)}{\Delta(0)}=\left[\left(\beta^{2}+\delta^{2}\right)\left[\alpha_{\mathbf{B}}\left(\alpha_{\mathbf{A}}+\alpha_{\mathbf{X}}\right)+\frac{\alpha_{\mathrm{A}} \alpha_{\mathbf{X}}}{f}\right]+\beta\left(\alpha_{\mathbf{A}}+\alpha_{\mathbf{X}}\right)\right]^{-1} \\
& \times\left[\begin{array}{c}
\delta\left\{\frac{\alpha_{\mathrm{A}} \alpha_{\mathrm{X}}}{f}+\alpha_{\mathrm{B}}\left(\alpha_{\mathrm{A}}+\alpha_{\mathrm{X}}\right)\right\} \\
\beta\left\{\frac{\alpha_{\mathrm{A}} \alpha_{\mathrm{X}}}{f}+\alpha_{\mathrm{B}}\left(\alpha_{\mathrm{A}}+\alpha_{\mathrm{X}}\right)\right\} \\
\beta\left(\alpha_{\mathrm{A}}+\alpha_{\mathrm{X}}\right) / 2 \\
\frac{\beta \alpha_{\mathrm{X}}}{2}
\end{array}\right] \\
& \mathbf{A}=\lim _{p \rightarrow-a}(p+a) \tilde{\mathbf{X}}(p)=\frac{-\mathbf{g}(-a)}{a(b-a)\left\{(c-a)^{2}+s^{2}\right\}} \\
& \mathbf{B}=\lim _{p \rightarrow-b}(p+b) \tilde{\mathbf{X}}(p)=\frac{-\mathbf{g}(-b)}{b(a-b)\left\{(c-b)^{2}+s^{2}\right\}} .
\end{aligned}
$$

Once $\mathbf{A}, \mathbf{B}$, and $\mathbf{E}$ are fixed, the vectors $\mathbf{C}$ and $\mathbf{D}$ are readily obtainable from the initial conditions on $\mathbf{X}$ and on its time derivative; i.e.,

$$
\mathbf{C}=\mathbf{X}(0)-\mathbf{A}-\mathbf{B}-\mathbf{E} \quad \text { and } \quad \mathbf{D}=a \mathbf{A}+b \mathbf{B}+c \mathbf{C}+\mathbf{X}^{\prime}(0) .
$$

\section{ACKNOWLEDGMENT}

The authors are indebted to Professors R. G. Bryant and B. M. Fung for their interest in this problem and valuable insights from our stimulating discussions with them.

\section{REFERENCES}

1. S. H. Koenig, R. G. Bryant, K. Hallenga, AND G. A. JACOB, Biochemistry 17, 4348 (1978).

2. H. T. EDZES AND E. T. SAMULSKI, J. Magn. Reson. 31, 207 (1978). 
3. H. T. Edzes and E. T. SAMUlSki, Nature 265, 521 (1977).

4. B. M. Fung AND T. W. MCGaUghy, J. Magn. Reson. 39, 413 (1980).

5. J. GRad AND R. G. BRyant, J. Magn. Reson. 90, 1 (1990).

6. S. D. Wol.fF and R. S. BaI.ABan, Magn. Reson. Med. 10, 135 (1989).

7. J. ENG, T. L. CECKLER, AND R. S. BaLABAN, Magn. Reson. Med. 17, 304 (1991).

8. J. Grad, D. MENDELSON, F. Hyder, AND R. G. Bryant, Magn. Reson. Med. 17, 452 (1991).

9. J. Grad, D. MfNDFlson, F. Hydfr, AND R. G. Bryant, J. Magn. Reson. 86, 416 (1990).

10. H. N. YEUNG, Abstracts of the Society of Magnetic Resonance in Medicine, 10th Annual Meeting, San Francisco, p. 174, 1991.

11. S. D. WOLFF AND R. S. Balaban, Radiology 179, 133 (1991).

12. S. D. WolfF, S. Chesnick, J. A. Frank, K. O. Lim, and R. S. Balaban, Radiology 179, 623 (1991).

13. H. N. YeUNG AND A. M. AISEN, Radiology 183, 209 (1992).

14. X. WU, J. Magn. Reson. 94, 186 (1991).

15. H. C. TORREY, Phys. Rev. 76, 1059 (1949).

16. S. D. Swanson, J. Magn. Reson. 95, 615 (1991).

17. D. C. LOOK AND D. R. LOCKER, Rev. Sci. Instrum. 41, 250 (1970).

18. M. SPIGORELLI, Department of Pharmacology, University of Michigan, private communication, 1991. 